\title{
Epigenetic treatment-mediated modulation of PD-L1 predicts potential therapy resistance over response markers in myeloid malignancies: A molecular mechanism involving effectors of $\mathrm{PD}-\mathrm{L} 1$ reverse signaling
}

\author{
HONGBIN LIU ${ }^{1}$, YUNSHAN HU ${ }^{1}$, RAFAEL RIMOLDI ${ }^{1}$, CHLOE VON HAGT ${ }^{1}$, EDMUND W.C. KHONG $^{1}$, \\ NORA LEE ${ }^{2}$, SHAUN FLEMMING ${ }^{3,4}$, ANDREW SPENCER ${ }^{3,4}$ and ANTHONY E. DEAR ${ }^{1,3}$ \\ ${ }^{1}$ Department of Medicine, Eastern Health Clinical School, Monash University; ${ }^{2}$ Department of Haematology, \\ Eastern Health Clinical School, Box Hill Hospital, Melbourne, Victoria $3128 ;{ }^{3}$ Australian Centre for Blood Diseases, \\ Monash University; ${ }^{4}$ Department of Haematology, Alfred Hospital, Melbourne, Victoria 3004, Australia
}

Received July 6, 2018; Accepted November 15, 2018

DOI: $10.3892 / \mathrm{ol} .2018 .9841$

\begin{abstract}
Recent evidence suggests an association exists between resistance to epigenetic therapy (EGT) and the expression of programmed cell death protein 1 (PD-1) and programmed death-ligand 1 (PD-L1) in myeloid malignancies. Biomarkers are required to predict resistance to EGT in myeloid malignancies, which together with the delineation of associated molecular mechanisms, may provide additional understanding for novel treatment strategies when investigating resistance to EGT. The present study aimed to investigate the in vivo effects of EGT on the expression of PD-1, PD-L1 and orphan nuclear receptor NUR77 with clinical responses in patients with myeloid malignancies. In addition, in vitro and in vivo characterization of the effects of EGT on the expression of NF- $\mathrm{KB}$ and Bcl-xL, potential downstream targets of PD-L1 reverse signaling, were evaluated to determine components of the molecular mechanism responsible for these effects. The in vivo effects of EGT on the expression of PD-1, PD-L1 and a previously identified molecular marker of response to EGT, NUR77 was characterized in peripheral blood mononuclear cells (PBMC) from patients with myelodysplastic syndrome (MDS) and acute myeloid leukemia (AML) treated with either azacytidine (Aza) alone or a combination of Aza and the histone deacetylase inhibitor (HDACi) LBH-589 during the first cycle of therapy. The correlation of clinical responses to treatment with EGT with the expression of PD-1, PD-L1 and NUR77 demonstrated that the induction of PD-L1
\end{abstract}

Correspondence to: Professor Anthony E. Dear, Department of Medicine, Eastern Health Clinical School, Monash University, 5 Arnold Street Box Hill, Melbourne, Victoria 3128, Australia E-mail: anthony.dear@monash.edu

Key words: epigenetic, programmed cell death protein 1, myelodysplasia, myeloid leukemia, biomarker
mRNA levels was associated with resistance to EGT despite the concurrent augmentation of NUR77 expression. The characterization of potential downstream effector molecules of reverse PD-L1 signaling identified EGT-mediated induction of Bcl-xL and NF- $\kappa \mathrm{B}$ mRNA expression in vitro and in vivo, suggesting a potential anti-apoptotic molecular mechanism was responsible for PD-L1-mediated resistance to EGT. Taken together, these observations suggest that enhanced PD-L1 expression may confer resistance to EGT over known EGT response markers in myeloid malignancies, and provides a potential molecular mechanism involving the modulation of effectors of PD-L1 reverse signaling, which may in-part, be responsible for these effects.

\section{Introduction}

Epigenetic therapy (EGT), specifically with hypomethylating agents (HMA) either alone or in combination, continues to be successfully used in the treatment of high risk myelodysplastic syndrome (MDS) and elderly acute myeloid leukemia (AML) although resistance is a frequent and ultimately near universal outcome (1). Mechanisms of de-novo resistance to EGT in the setting of MDS and AML are incompletely understood however recent studies have identified diverse molecular mechanisms including integrin $\alpha 5$-mediated hematopoietic progenitor cell quiescence (2), increased RNA 5-methylcytosine active chromatin (3), elevated BCL2L10 expression (4) and Stat3/5 signalling (5) as contributing factors.

MDS and AML are often associated with endogenous immune compromise and overexpression of immune checkpoint molecule expression (6). Identification of EGT-mediated upregulation of genes associated with tumour immune evasion including immune checkpoint pathway-related molecules programmed cell death protein 1 (PD-1), programmed death-ligand 1 (PD-L1) and programmed death ligand 2 (PD-L2), may contribute to the acquired EGT resistance phenotype through $\mathrm{T}$ cell exhaustion $(6,7)$. In addition 
EGT-mediated induction of tumour cell PD-L1 expression may contribute to enhanced PD-L1 reverse signalling facilitating anti-apoptotic effects in tumour cells and emergence of disease resistance (8).

The recent development of immune checkpoint inhibitor therapy, successfully trialled in solid tumours and more recently in lymphoid haematological malignancies $(6,7)$, may afford therapeutic benefit in the setting of EGT-mediated immune evasion based resistance should these patients be identifiable.

Despite identification of several putative markers of early response to $\mathrm{EGT}(3,5,9,10)$ characterisation of biomarkers of acquired resistance to EGT in myeloid malignancies are required together with delineation of the associated molecular mechanisms.

Our study aimed to correlate the in vivo effects of EGT on expression of PD-1, PD-L1 and orphan nuclear receptor NUR77, previously identified as a key molecule in the response to EGT (11), with clinical response in patients with myeloid malignancies. In addition, in vitro and in vivo characterisation of the effects of EGT on NF-кB and Bcl-xL expression, potential downstream targets of PD-L1 reverse signalling, was evaluated to delineate possible components of the molecular mechanism responsible for these effects which may inform on novel treatment strategies in the setting of resistance to EGT.

\section{Materials and methods}

Cell culture. KG-1 leukemia cells were cultured in IMDM (Gibco; Thermo Fisher Scientific, Inc., Waltham, MA, USA) containing $20 \%$ heat inactivated fetal calf serum (Gibco; Thermo Fisher Scientific, Inc.) and kept in a $5 \% \mathrm{CO}_{2}$ incubator at $37^{\circ} \mathrm{C}$. Cells were split into 6 -well plates with $2 \times 10^{6}$ cells per well. Agents: Azacytidine (Aza) (Sigma, Australia) and the histone deacetylase inhibitors (HDACi) Panobinostat (LBH-589) (LBH) (Sapphire Bioscience Pty Ltd., Sydney, Australia) and MCT-3 (12) were added to plates for $24 \mathrm{~h}$. Aza was dissolved in $\mathrm{H}_{2} \mathrm{O}$ with $0.2 \%$ acetic acid and used at a final concentration of $1.0 \mu \mathrm{M}$. LBH-589 and MCT-3 were dissolved in $1 \%$ DMSO and used at a final concentration of $20 \mathrm{nM}$ and $0.5 \mu \mathrm{M}$ respectively.

Patient and healthy control blood samples. Mono-nuclear cell fractions were obtaining by ficoll density gradient centrifugation of peripheral blood samples taken from patients enrolled in low risk studies 357/14 and LR63/2015 with MDS/AML treated with Aza alone ( $\mathrm{n}=9), 75 \mathrm{mg} / \mathrm{m}^{2}$ for 7 days of a 28 day cycle between February 2015 and January 2017 and from a phase Ib/II clinical trial $(n=4)$ of a 5 day schedule of Aza, $75 \mathrm{mg} / \mathrm{m}^{2}$ followed by LBH in high-risk MDS or AML patients between January 2010 and January 2012 (13) and healthy controls aged 25-50 years old) $(n=5)$. Peripheral blood samples were collected from patients at screening (prior to treatment) and at time points (days 5-28) during the first cycle of treatment. The open-label, phase Ib/II study (13) was conducted at three centres: Alfred Hospital, Melbourne, Australia; Princess Alexandra Hospital, Brisbane, Australia; and Austin Hospital, Melbourne, Australia. Low risk studies 357/14 and LR63/2015 were conducted at the Alfred Hospital, Melbourne, Australia and Box Hill Hospital, Melbourne, Australia.
The open-label, phase Ib/II study (13) was approved by the Alfred Hospital Ethics Committee, Melbourne, Australia on 15/07/2009, ethics approval number AH189/09, and performed in accordance with the principles of independent Human Research and Ethics Committees, and registered with the Australian and New Zealand Clinical Trials Registry (ACTRN12610000924055). Low risk study 357/14 was approved by Alfred Health Ethics Committee on 20/8/2014 and low risk study LR63/2015 was approved by Eastern Health Ethics Committee, Melbourne, Australia on 27/07/2015. All participating patients and healthy controls were required to provide written informed consent for participation in the studies.

Growth inhibition assay. KG-1 cells in log phase were plated at a density of $0.2 \times 10^{6}$ in $2 \mathrm{ml}$ of medium. Cells were harvested at $24 \mathrm{~h}$. Cell viability was assessed using $0.4 \%$ trypan blue staining immediately after culture. Black staining cells were considered as non-viable cells and unstained bright cells as viable. Cell growth $(\%)=($ the total number of viable cells at $24 \mathrm{~h}$-the total number of viable cells at the beginning of the experiment/the total number of viable cells at the beginning of the experiment) as previously described (9). All experiments were repeated a minimum of 3 times with averages displayed graphically.

RNA extraction. TRIzol (Invitrogen; Thermo Fisher Scientific, Inc.) was used to extract RNA from KG-1 cells treated with Aza, LBH or MCT-3 or a combination of Aza+LBH. TRIzol was also used to extract RNA from the mono-nuclear cell fractions of blood samples from patients with MDS/AML treated with Aza alone, $75 \mathrm{mg} / \mathrm{m}^{2}$ for 7 days of a 28 day cycle or from a phase $\mathrm{Ib} / \mathrm{II}$ clinical trial of a 5 day schedule of Aza followed by LBH in high-risk MDS or AML patients (13) and healthy controls. A single mRNA sample for each time point per patient was available for extraction.

$R T-q P C R$. Reverse Transcription of KG-1 cell and patient and healthy control mRNA was performed using a $20 \mu 1$ reaction mix containing dNTPs (100 mM), MultiScribe Reverse Transcriptase (50 U/ $\mu \mathrm{l})$, RT buffer (10X), RNase Inhibitor $(20 \mathrm{U} / \mu \mathrm{l})$, nuclease free water (Invitrogen; Thermo Fisher Scientific, Inc.), primer and KG-1 cell, patient or healthy control RNA samples. RT-qPCR analysis of KG-1 cell, patient and healthy control PD-1, PD-L1, NUR77, Bcl-xL and NF-KB mRNA expression was performed in duplicate using Sensifast SYBR No-Rox kit (Bioline, London, UK). Reaction volumes of $20 \mu 1$ contained 2X Sensifast SYBR no-rox mix, nuclease-free water and RT reaction product (either KG-1 cell or patient/healthy control sample). Each PCR run also included wells of no template control (NTC). A melting point dissociation curve generated by the instrument (Applied Biosystems 7500 Real-Time PCR System; Thermo Fisher Scientific, Inc.) was used to confirm that only a single product was present. The fluorescence data were quantitated using the threshold cycle $\left(C_{t}\right)$ value (14). Data was normalized to actin and presented as the fold change compared with the pre-treatment screen sample.

PCR primers. Forward and reverse primer sequences for: PD-1: Forward 5'-GGAAACCCCTCCACCTTTA-3' and 

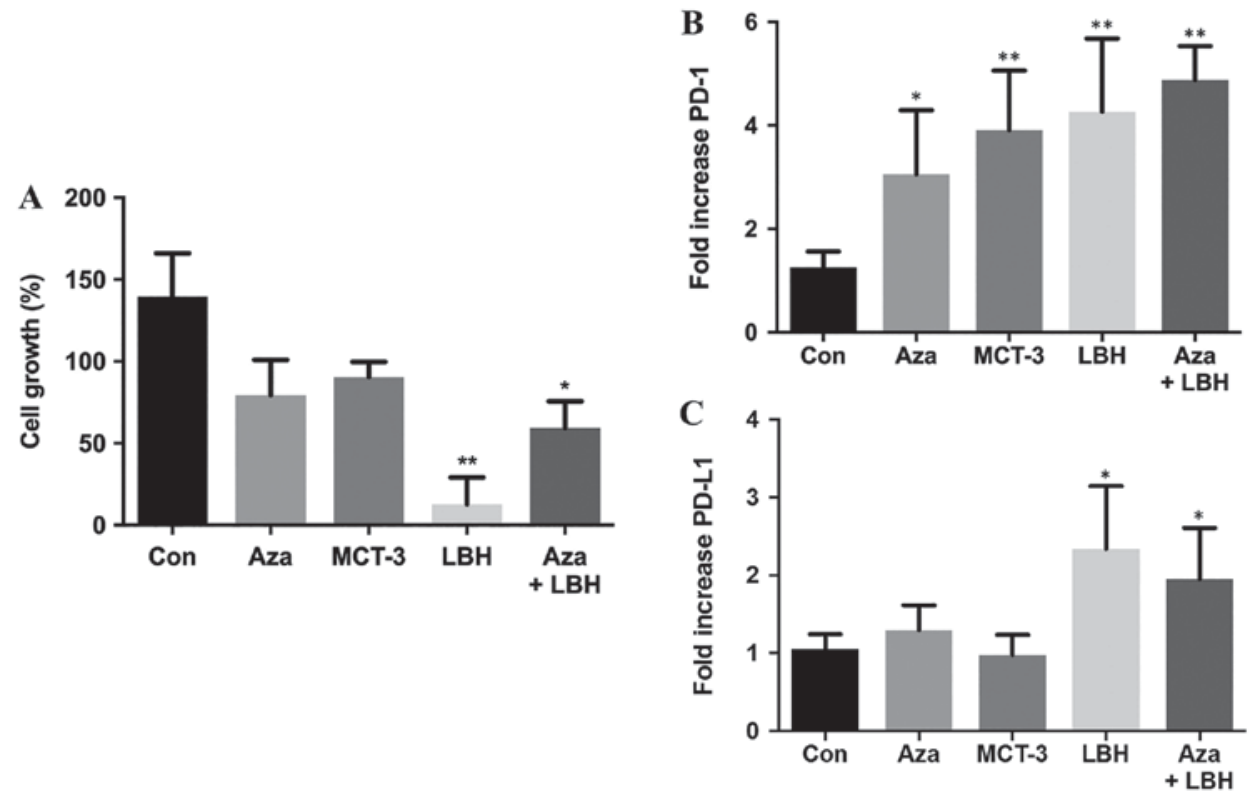

Figure 1. In vitro effects on cell growth, mRNA expression levels of PD-1 and PD-L1 of Aza, LBH-589, MCT-3 or a combination of Aza and LBH589 in KG-1 cells. (A) In vitro effects on cell growth in KG-1 cells untreated (Con) or treated for $24 \mathrm{~h}$ with Aza, LBH-589 (LBH), MCT-3 or Aza+LBH as assessed by trypan blue staining. $\mathrm{n}=3$. In vitro mRNA expression of (B) PD-1 and (C) PD-L1 mRNA in KG-1 cells untreated (Con) and treated for 24-h treatment with Aza, MCT-3, LBH or a combination of Aza+LBH. ${ }^{*} \mathrm{P}<0.05$ vs. con, ${ }^{* *} \mathrm{P}<0.01$ vs. con, $\mathrm{n}=3-4$. PD-1, programmed cell death protein 1; PD-L1, programmed death-ligand 1; Aza, azacytidine; Con, control.

reverse 5'-TCTGCCTGCCCGCTTACT-3'; PD-L1: Forward 5'-TGGCATTTGCTGAACGCATTT-3' and reverse 5'-TGC AGCCAGGTCTAATGTTTT-3'; NUR77: Forward 5'-GCT GCAGAATGACTCCACC-3' and reverse 5'-ACAGCACTG GGCTTA-3'; NF-кB: Forward 5'-CAGGAAGATGTGGAGG AT-3' and reverse 5'-TGTCGTGCTCCACAGCCAGGT-3'; Bcl-xL: Forward 5'-TTGGACAATGGACTGGTTGA-3' and reverse 5'-GTAGAGTGGATGGTCAGTG-3'; and actin: Forward 5'-GACAGGATGCAGAAGGAGATTACT-3' and reverse 5'-TGATCCACATCTGCTGGAAGGT-3'.

Statistical methods. Results of in vitro studies and combined patient in vivo studies were expressed as means \pm standard error of the mean (SEM), and analyzed using GraphPad Prism 5 software (GraphPad Software, Inc., La Jolla, CA, USA), using unpaired t-tests for two-group comparisons and one-way analysis of variance and Dunnett's post hoc (ANOVA) for three or more group comparisons. P-value of $<0.05$ was considered to be statistically significant.

Methodological evaluation of results of duplicate RT-qPCR analysis of individual patient, single time point mRNA samples were presented as mean fold change compared to screen utilizing the $2^{-\Delta \Delta \mathrm{Cq}}$ method (14).

\section{Results}

Effect of Aza, LBH-589 and MCT-3 on leukemic cell growth, $P D-1$ and PD-L1 mRNA expression in KG-1 cells. Single agent treatment with Aza, MCT-3 and LBH-589 decreased KG-1 leukemic cell growth with LBH-589 showing significant inhibition of cell growth. Combination Aza+LBH-589 treatment also resulted in significant attenuation of cell growth (Fig. 1A). Single agent treatment with Aza, MCT-3 and LBH-589 resulted in significant induction of PD-1 mRNA expression whilst combination treatment with Aza+LBH-589 also demonstrated significant induction of PD-1 mRNA expression (Fig. 1B). Single agent treatment with Aza demonstrated modest induction of PD-L1 mRNA expression whilst LBH-589 treatment resulted in significant induction of PD-L1 mRNA expression and combination treatment with Aza+LBH-589 also demonstrated significant induction of PD-L1 mRNA expression (Fig. 1C).

PD-1, PD-L1 and NUR77 mRNA expression profiles from $P B M C$ 's of patients treated with Aza alone or Aza in combination with LBH589. Expression levels of PD-1, PD-L1 and NUR77 mRNA were determined at screening (prior to treatment commencement, day 0) and at designated time points during 25-28 day first cycle of treatment. Non-responders demonstrated a $>2$ fold increase in PD-L1 expression over any other time point after treatment commenced during the first cycle of treatment (Fig. 2A and Table I) whilst responders, apart from patients 001 and 006, demonstrated no increase in PD-L1 expression (Fig. 2B and Table I). No correlation of PD-1 expression with response was identified in individual patient analysis whilst paradoxically 4 of 6 non-responders also had induction of NUR77 expression, a previously identified putative marker of response to EGT in MDS (Fig. 2A and Table I). Comparison of PD-L1 expression from combined first cycle time points (screen, day 14, 21 and 28) of healthy controls, responders and non-responders demonstrated significant induction of PD-L1 expression in non-responders over both healthy controls and responders (Fig. 3). Responses were defined according to international working group criteria for AML and MDS (15).

In vitro and in vivo expression of $N F-\kappa B$ and $B c l-x L$ in $A z a$, LBH-589, MCT-3 or a combination of Aza and LBH-589treated 


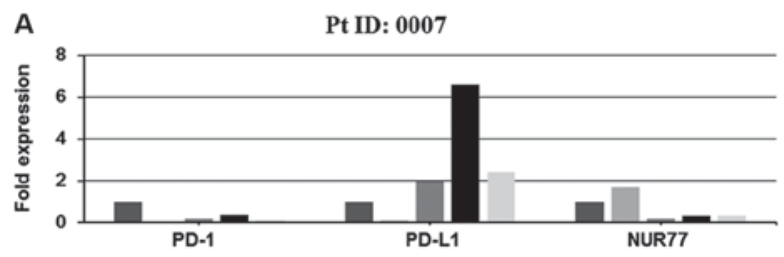

Day: 0 Day: $5 \square$ Day: 15 Day: 19 Day: 25

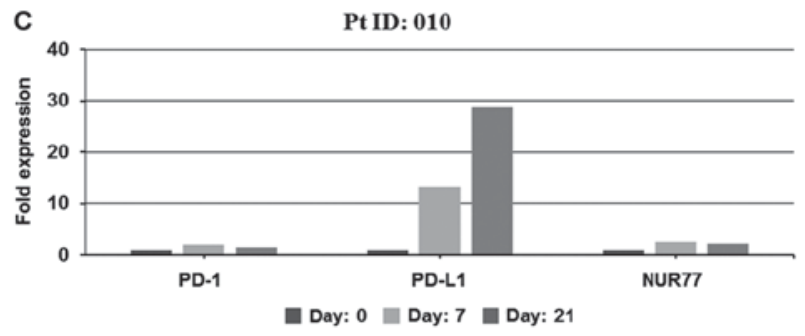

E

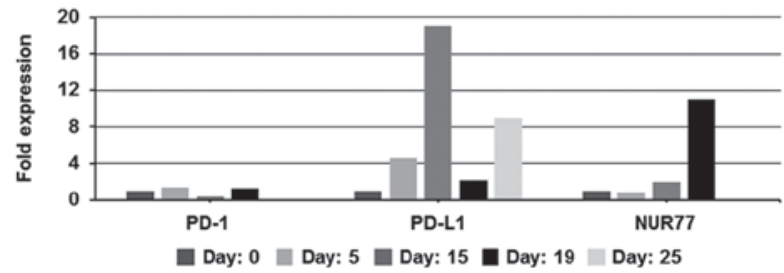

B

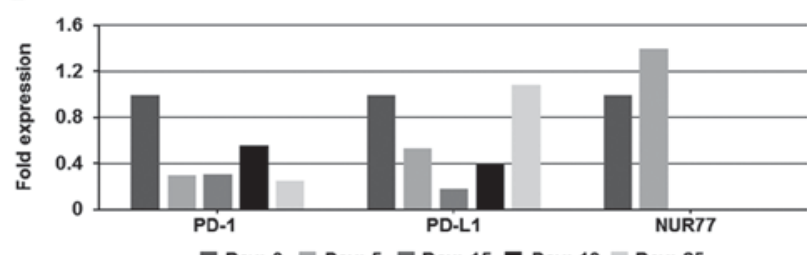

Day: 0 Day: 5 Day: 15 Day: 19 Day: 25

D

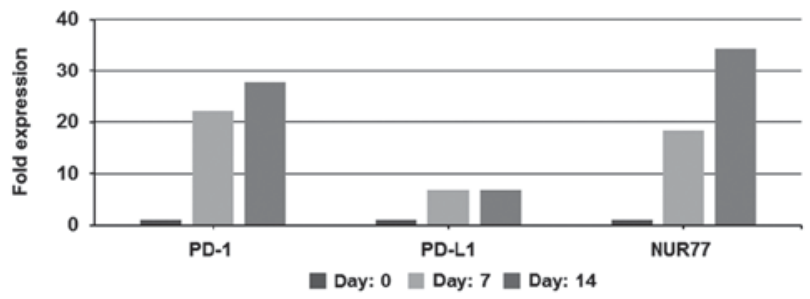

$\mathbf{F}$

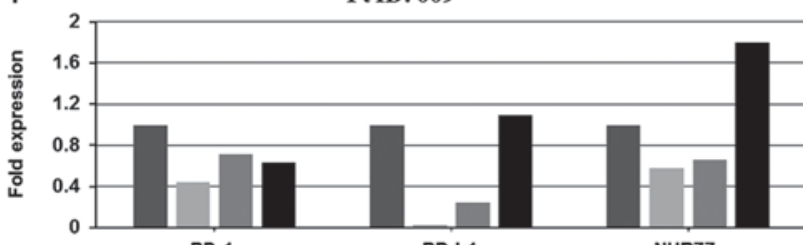

PD-1
Pt ID: 009

Pt ID: 003

Day: 0 Day: 7 Day: 21 Day: 28

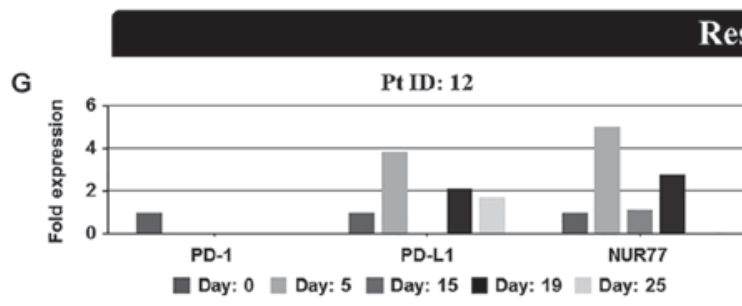

I
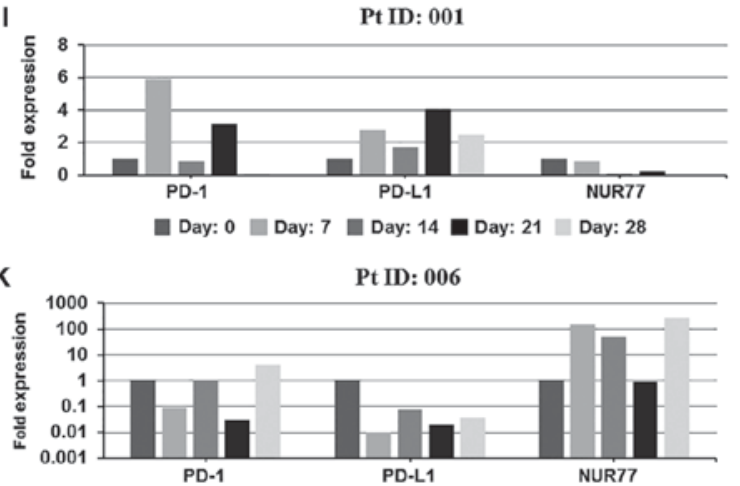

Day: 0 Day: 7 Day: 14 Day: 21 Day: 28

M

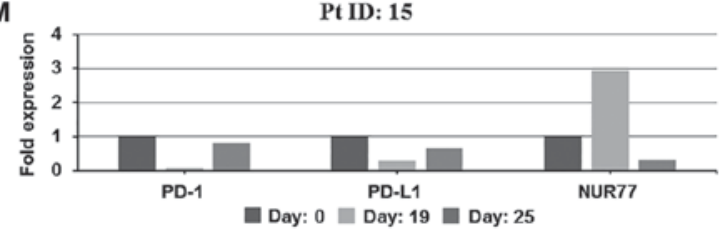

Responders

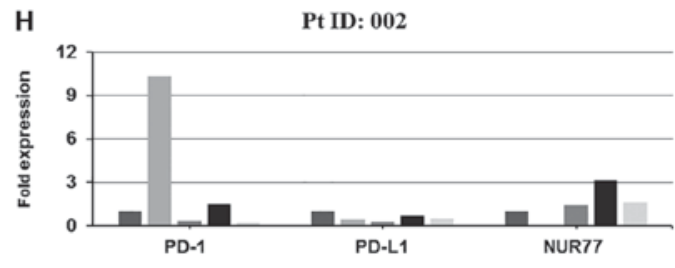

Day: 0 Day: 7 Day: 14 Day: 21 Day: 28
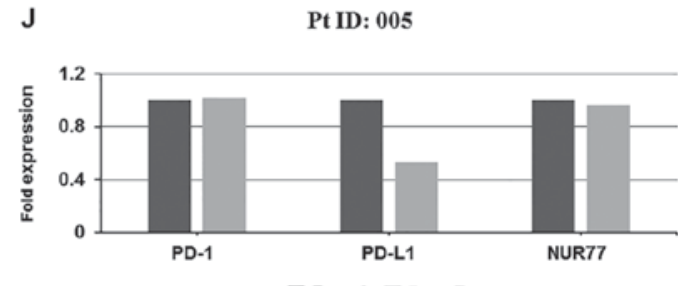

Day: 0 Day: 7

L

Pt ID: 007

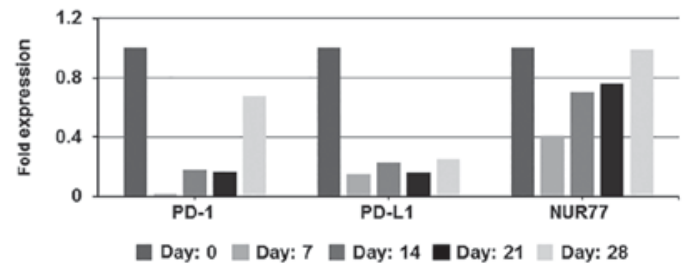

Figure 2. In vivo mRNA expression profiles at screening and during cycle 1 of PD-1, PD-L1 and NUR77 from PBMC's of individual non-responder and responder patients treated with Aza alone or a combination of Aza and LBH589. Non-responders: (A) patient 0007 (resistant), (B) patient 0021 (resistant), (C) patient 010 (resistant), (D) patient 003 (resistant), (E) patient 0013 [stable disease (SD)], (F) patient 009 [progressive disease (PD)]. Day: 0=screening sample, Days 5-28=samples taken on specific day post screening. Responders: (G) patient 12 [partial response (PR)], (H) patient 002 (PR), (I) patient 001 [complete response (CR)], (J) patient 005 (CR), (K) patient $006(\mathrm{CR}),(\mathrm{L})$ patient 007 (CR), and (M) patient 015 [not evaluated (NE)]. Clinical response to treatment determined at 1,3 and 6 months. Responses were defined according to international working group criteria for AML and MDS (15). Results for patient 006 expression levels are presented using a log scale. PD-1, programmed cell death protein 1; PD-L1, programmed death-ligand 1; PBMC, peripheral blood mononuclear cells; Aza, azacytidine; AML, acute myeloid leukemia; MDS, myelodysplastic syndrome. 
Table I. Clinical response data and correlation with PD-1, PD-L1 and NUR77 mRNA expression at days 5-28.

\begin{tabular}{|c|c|c|c|c|c|c|}
\hline Patient ID & $\begin{array}{c}\text { Sex/age } \\
\text { (years) }\end{array}$ & Diagnosis/treatment & $\begin{array}{c}\text { Best } \\
\text { response }\end{array}$ & $\begin{array}{c}\text { PD-1 } \\
\text { induction }^{\mathrm{a}}\end{array}$ & $\begin{array}{l}\text { PD-L1 } \\
\text { induction }^{\mathrm{a}}\end{array}$ & $\begin{array}{c}\text { NUR77 } \\
\text { induction }^{\mathrm{a}}\end{array}$ \\
\hline 0007 & M 58 & $\mathrm{AML}^{\mathrm{b}}$ & Resistant & No & Yes & No \\
\hline 0021 & F 78 & $\mathrm{AML}^{\mathrm{b}}$ & Resistant & No & Yes & No \\
\hline 003 & M 75 & $\mathrm{AML}^{\mathrm{c}}$ & Resistant & Yes & Yes & Yes \\
\hline 010 & F 65 & $\mathrm{AML}^{\mathrm{c}}$ & Resistant & Yes & Yes & Yes \\
\hline 0013 & M 79 & $\mathrm{MDS}^{\mathrm{b}}$ & $\mathrm{SD}$ & Yes & Yes & Yes \\
\hline 009 & F 89 & $\mathrm{MDS}^{\mathrm{c}}$ & PD & No & Yes & Yes \\
\hline 12 & F 59 & $\mathrm{MDS}^{\mathrm{c}}$ & PR & No & No & Yes \\
\hline 002 & M 60 & $\mathrm{AML}^{\mathrm{c}}$ & PR & Yes & No & Yes \\
\hline 001 & M 45 & $\mathrm{MDS}^{\mathrm{c}}$ & $\mathrm{CR}$ & Yes & Yes & No \\
\hline 005 & M 78 & $\mathrm{MDS}^{\mathrm{c}}$ & $\mathrm{CR}$ & No & No & No \\
\hline 006 & F 90 & $\mathrm{MDS}^{\mathrm{c}}$ & $\mathrm{CR}$ & No & Yes & Yes \\
\hline 007 & M 64 & $\mathrm{AML}^{\mathrm{c}}$ & CR & No & No & No \\
\hline 15 & M 77 & $\mathrm{MDS}^{\mathrm{b}}$ & NE & No & No & Yes \\
\hline
\end{tabular}

${ }^{a}>2$ fold induction in gene expression following treatment commenced during cycle 1 (days 5-28). PD-1, programmed cell death protein 1 ; PD-L1, programmed death-ligand 1; NUR77, orphan nuclear receptor NUR77; MDS, myelodysplastic syndrome; AML, acute myeloid leukemia; CR, complete response; PR, partial response; SD, stable disease; PD, progressive disease; M, male; F, female; NE, not evaluated. ${ }^{b}$ Epigenetic treatment with azacytidine and LBH-589. ${ }^{\circ}$ Epigenetic treatment with azacytidine alone.

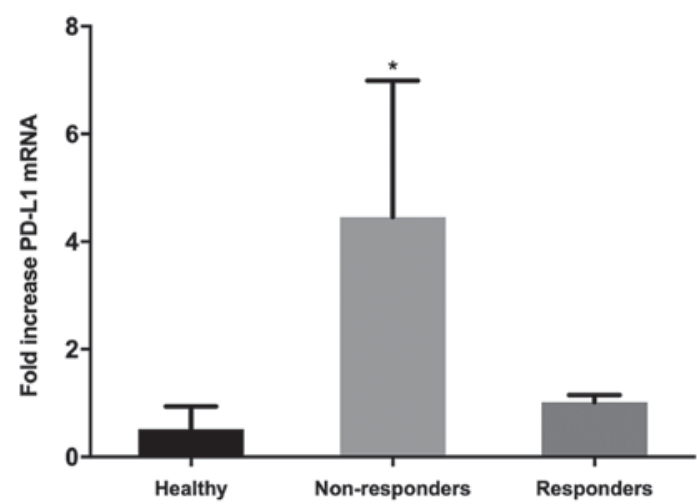

Figure 3. In vivo mRNA expression of PD-L1 from combined healthy controls, responders and non-responders during first cycle of treatment. PD-L1 mRNA expression was determined from PBMC's from combined healthy controls $(n=5)$, and combined EGT responders $(n=7)$ and non-responders $(n=6)$ during first cycle of treatment, ${ }^{*} \mathrm{P}<0.05$ healthy control vs. non-responders and non-responder vs. responder. Responses were defined according to international working group criteria for AML and MDS (15). PD-L1, programmed death-ligand 1; PBMC, peripheral blood mononuclear cells; EGT, epigenetic therapy; AML, acute myeloid leukemia; MDS, myelodysplastic syndrome..

$K G-1$ cells and in patients non-responsive and responsive to $E G T$. PD-L1 reverse signaling has recently been identified as a potential mechanism of EGT resistance in the setting of MDS treatment $(8,16-19)$. We investigated the in vitro and in vivo effect of EGT on potential down-stream effector molecules of reverse PD-L1 signaling. In vitro studies identified 2-8 fold induction of NF- $\kappa \mathrm{B}$ and $\mathrm{Bcl}-\mathrm{xL}$ mRNA expression in $\mathrm{KG}-1$ cells treated with single agent or combination EGT (Fig. 4A and B). Characterisation of Bcl-xL expression from two patients, one identified as non-responsive to EGT (Pt ID:010) and one identified as responding to EGT (Pt ID:007) identified significant upregulation of Bcl-xL mRNA expression in the patient resistant to EGT (Fig. 4C) together with unchanged or reduced levels of $\mathrm{Bcl}-\mathrm{xL}$ and $\mathrm{NF}-\kappa \mathrm{B}$ mRNA in the patient who responded to EGT (Fig. 4D and E).

\section{Discussion}

Treatment of myeloid malignancies with single or combination agent EGT has proven to be a significant advance in the management of these conditions (20). Response rates in the order of $50 \%$, delayed time to response, together with primary and acquired resistance to EGT have made identification of biomarkers of response and resistance, together with associated molecular mechanisms, increasingly important in the management of patients treated with EGT (20).

Recent evidence identifies EGT-mediated upregulation of genes associated with tumour immune evasion, including immune checkpoint pathway-related molecules PD-1, PD-L1 and PD-L2 in the setting of treatment of myeloid malignancies, which may contribute to the acquired EGT resistance phenotype through T cell exhaustion and enhanced PD-L1 reverse signaling $(6-8,18)$.

Our current study investigated the expression of immune checkpoint molecules PD-1 and PD-L1 together with the putative EGT response marker orphan nuclear receptor NUR77 $(9,11)$ in PBMC's from patients with myeloid malignancies treated with EGT and healthy controls and correlated these findings with clinical response. In addition we investigated the in vitro and in vivo effects of EGT on expression of potential downstream molecules of reverse PD-L1 signalling as a possible molecular mechanism for acquired resistance to EGT.

PD-L1 expression was increased in vitro in response to EGT and correlated with resistance to EGT irrespective of increased expression of the previously postulated marker of 

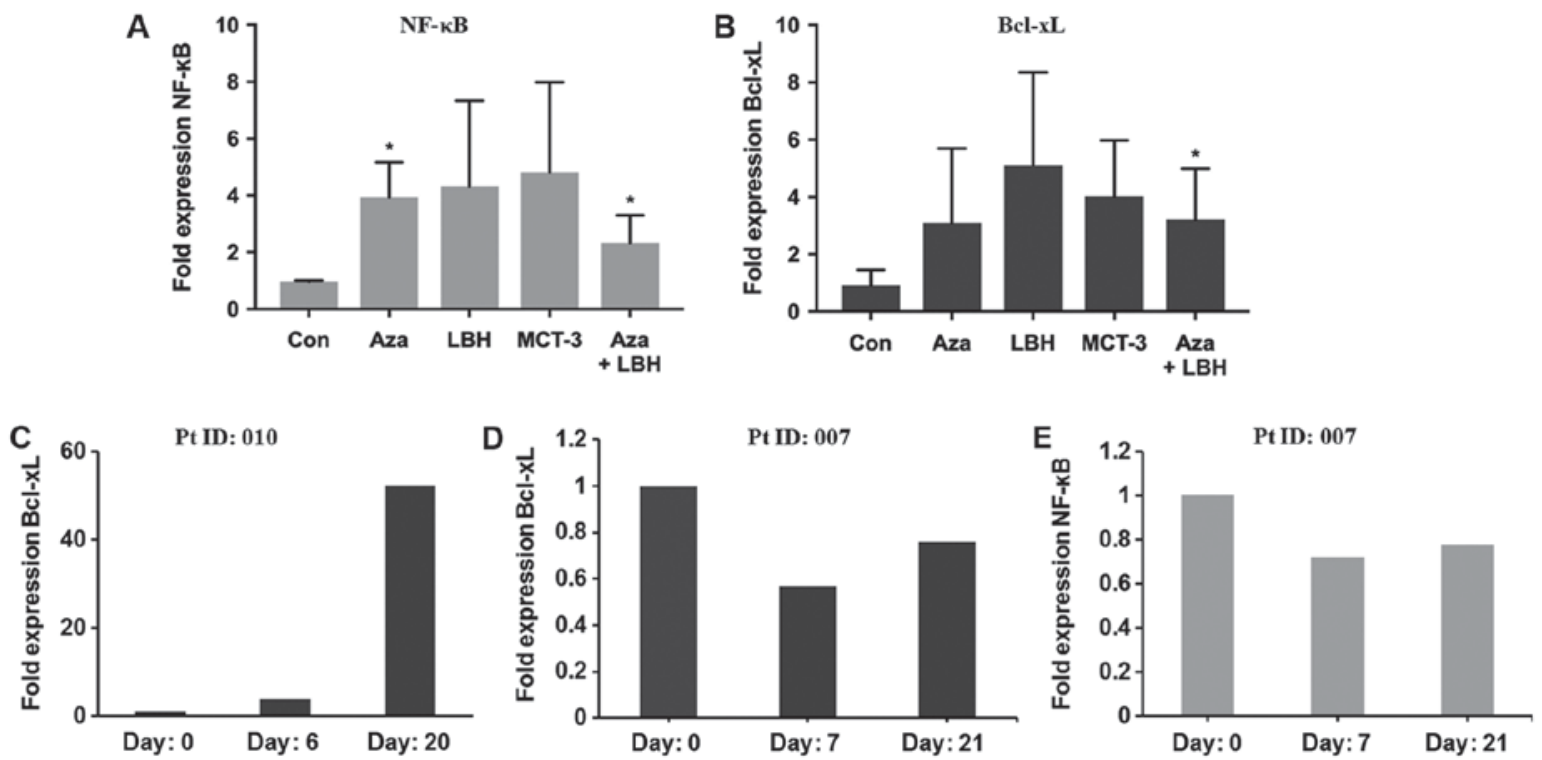

Figure 4. In vitro and in vivo mRNA expression levels of NF-kB and Bcl-xL in KG-1 cells treated with Aza, LBH-589, MCT-3 or a combination of Aza+LBH-589 and from PBMC's from a non-responder and responder patient. (A) NF-kB and (B) Bcl-xL mRNA expression in KG-1 cells untreated (Con) or treated for $24 \mathrm{~h}$ with Aza, LBH-589 (LBH), MCT-3 or Aza+LBH *P<0.05 Con vs. Aza and Aza+LBH (n=3) and (C) Bcl-xL mRNA expression in PBMC's from non-responsive patient 010 at day 0, 6 and day 20 (n=1). (D) Bcl-xL and (E) NF-kB mRNA expression in PBMC's from an EGT-responsive patient 007 at day 0,7 and day 21 $(\mathrm{n}=1)$. Responses were defined according to international working group criteria for AML and MDS (15). Aza, azacytidine; PBMC, peripheral blood mononuclear cells; EGT, epigenetic therapy; Con, control; AML, acute myeloid leukemia; MDS, myelodysplastic syndrome.

response to EGT, NUR77 suggesting enhanced PD-L1 expression and associated downstream molecular mechanisms may be a more potent phenotypic contributor to EGT outcomes than potential response biomarkers including NUR77. Whilst previous studies have demonstrated EGT is able to increase PD-L1 expression in MDS (16-18) and may correlate with poor responses to treatment (18) our study is the first to identify this effect independent of potential response marker expression. Whilst our in vivo observations support our in vitro findings a potential limitation of our study exists due to logistical constraints in obtaining chronologically identical patient blood samples for analysis during cycle 1. Ongoing studies to evaluate more complete data sets over multiple cycles of EGT will be undertaken to further refine the observations made in this pilot study.

The molecular mechanisms responsible for EGT resistance and loss of response in the setting of EGT-mediated upregulation of inhibitory checkpoint molecules may include enhanced immune evasion by leukemic cells through classical PD-1-PD-L1 interactions (6-8) together with recently postulated augmentation of tumour cell PD-L1 reverse signaling (8).

The contribution of tumour cell-intrinsic PD-L1 reverse signalling to disease pathogenesis has been recently investigated. Initial reports identified B7-H1 or PD-L1 as a ubiquitous anti-apoptotic receptor on cancer cells (19) whilst comprehensive evaluation of tumour intrinsic PD-L1 signalling in ovarian and melanoma models have demonstrated significant stimulatory effects on inflammation and tumour cell growth (21). Our studies evaluated potential downstream targets of PD-L1 reverse signalling including the apoptosis and inflammation associated genes Bcl-xL and NF- $\mathrm{KB}$. EGT-mediated induction of Bcl-xL and NF- $\mathrm{KB}$ gene expression both in vitro and in vivo in the setting of treatment resistance demonstrates that enhanced PD-L1 expression may correlate with induction of PD-L1 reverse signalling and upregulation of downstream targets which may contribute to tumour cell resistance to EGT. In support of these observations recent studies identify single or combination agent EGT treatment of the HL-60 leukemia cell line, known not to express PD-L1, as able to attenuate Bcl-xL expression, while concurrently enhancing expression of the putative response marker NUR77 (9) suggesting induction of PD-L1 expression together with enhanced PD-L1 reverse signalling and downstream effectors may be significant contributors to treatment resistance and tumour cell persistence. It is intriguing to speculate that whilst EGT in the setting of treatment for myeloid malignancies induces malignant cell apoptosis, as has been previously extensively documented in KG-1 cells $(22,23)$ and possibly supported by our observations of reduced KG-1 cell growth with in vitro EGT, simultaneous anti-apoptotic effects mediated by induction of PD-L1 expression together with enhanced PD-L1 reverse signalling and upregulation of Bcl-xL may contribute to malignant cell persistence and ultimately EGT failure.

EGT has recently been postulated to potentially enhance and/or re-sensitize solid and haematological malignancies to the effects of novel anti-PD-1/PD-L1 immunotherapeutics via enhanced tumour neoantigen expression (7) and/or via upregulation of expression of double stranded endogenous retroviral elements (dsERV's), subsequent viral defense gene expression and interferon (IFN) production, leading to cellular apoptosis and reduced cellular proliferation (24-26).

A so-called 'double edged sword effect' of EGT however arises from potentially concurrent therapy-mediated induction of inhibitory checkpoint molecule expression such as PD-L1 which may contribute to enhanced secondary resistance to EGT in leukemia and MDS via mechanisms including reverse PD-L1 signaling which we have investigated during this study. 
Whilst potentially problematic, EGT-mediated upregulation of inhibitory immune checkpoint molecule expression may be able to be exploited though identification of patients with EGT-mediated upregulation of inhibitory immune checkpoint molecules including PD-L1 and therapeutic intervention with anti-PD-1/PD-L1 therapy; a theory currently the subject of several clinical trials (7). In addition characterization of PD-L1 expression may be an important marker of acquired EGT resistance and anti-PD-1/PD-L1 therapy response in this patient population.

Together our observations suggest enhanced PD-L1 expression may herald resistance to EGT over known markers of response to EGT in myeloid malignancies and provide a potential molecular mechanism involving modulation of effectors of PD-L1 reverse signaling which may, in-part, be responsible for these effects. Larger prospective studies designed to formally evaluate the contribution of PD-L1 expression to acquired EGT resistance will establish the potential application of this molecule as a clinical biomarker and therapeutic target in the setting EGT resistance.

\section{Acknowledgements}

The abstract was presented as a poster presentation at the 30th Lorne Cancer Conference 2018 Feb 8-11 2018 in Lorne, Victoria, Australia.

\section{Funding}

No funding was received.

\section{Availability of data and materials}

The datasets used and/or analyzed during the current study are available from the corresponding author on reasonable request.

\section{Authors' contributions}

HL, YH, RR, CvH, EWCK, NL, and SF assisted with analysis, interpretation and presentation of data. AS was instrumental in conceiving and designing critical elements of the clinical trial utilized in this study. AED assisted with analysis and interpretation of data and made a significant intellectual contribution to the study. All authors contacted read and approved the final manuscript.

\section{Ethics approval and consent to participate}

The open-label, phase Ib/II study (13) was approved by the Alfred Hospital Ethics Committee on 15/07/2009, ethics approval number AH189/09 and was performed in accordance with the principles of independent human research and ethics committees, and registered with the Australian and New Zealand Clinical Trials Registry (ACTRN12610000924055). Low risk study 357/14 was approved by Alfred Health Ethics Committee (AHEC) on 20/8/2014 and low risk study LR63/2015 was approved by Eastern Health Ethics Committee (EHEC) on 27/07/2015. All participating patients and healthy controls were required to provide written informed consent for participation in the studies.

\section{Patient consent for publication}

Not applicable.

\section{Competing interests}

The authors declare that they have no competing interests.

\section{References}

1. Yun S, Vincelette ND, Abraham I, Robertson KD, Fernandez-Zapico ME and Patnaik MM: Targeting epigenetic pathways in acute myeloid leukemia and myelodysplastic syndrome: A systematic review of hypomethylating agents trials. Clin Epigenetics 8: 68, 2016.

2. Unnikrishnan A, Papaemmanuil E, Beck D, Deshpande NP, Verma A, Kumari A, Woll PS, Richards LA, Knezevic K, Chandrakanthan $\mathrm{V}$, et al: Integrative genomics identifies the molecular basis of resistance to azacitidine therapy in myelodysplastic syndromes. Cell Rep 20: 572-585, 2017.

3. Cheng JX, Chen L, Li Y, Cloe A, Yue M, Wei J, Watanabe KA, Shammo JM, Anastasi J, Shen QJ, et al: RNA cytosine methylation and methyltransferases mediate chromatin organization and 5-azacytidine response and resistance in leukaemia. Nat Commun 9: 1163, 2018.

4. Vidal V, Robert G, Goursaud L, Durand L, Ginet C, Karsenti JM, Luciano F, Gastaud L, Garnier G, Braun T, et al: BCL2L10 positive cells in bone marrow are an independent prognostic factor of azacitidine outcome in myelodysplastic syndrome and acute myeloid leukemia. Oncotarget 8: 47103-47109, 2017.

5. MiltiadesP,LamprianidouE,VassilakopoulosTP,PapageorgiouSG, Galanopoulos AG, Kontos CK, Adamopoulos PG, Nakou E, Vakalopoulou S, Garypidou V, et al: The Stat $3 / 5$ signaling biosignature in hematopoietic stem/progenitor cells predicts response and outcome in myelodysplastic syndrome patients treated with azacitidine. Clin Cancer Res 22: 1958-1968, 2016.

6. Daver N, Boddu P, Garcia-Manero G, Yadav SS, Sharma P, Allison J and Kantarjian H: Hypomethylating agents in combination with immune checkpoint inhibitors in acute myeloid leukemia and myelodysplastic syndromes. Leukemia 32: 1094-1105, 2018

7. Wolff F, Leisch M, Greil R, Risch A and Pleyer L: The double-edged sword of (re)expression of genes by hypomethylating agents: From viral mimicry to exploitation as priming agents for targeted immune checkpoint modulation. Cell Commun Signal 15: 13, 2017.

8. Granier C, De Guillebon E, Blanc C, Roussel H, Badoual C, Colin E, Saldmann A, Gey A, Oudard S and Tartour E: Mechanisms of action and rational for the use of checkpoint inhibitors in cancer. ESMO Open 2: e000213, 2017.

9. Liu HB, Urbanavicius D, Tan P, Spencer A and Dear AE: Mechanisms and potential molecular markers of early response to combination epigenetic therapy in patients with myeloid malignancies. Int J Oncol 45: 1742-1748, 2014.

10. Lübbert M, Ihorst G, Sander PN, Bogatyreva L, Becker H, Wijermans PW, Suciu S, Bissé E and Claus R: Elevated fetal haemoglobin is a predictor of better outcome in MDS/AML patients receiving 5-aza-2'-deoxycytidine (Decitabine). Br J Haematol 176: 609-617, 2017.

11. Zhou L, Ruvolo VR, McQueen T, Chen W, Samudio IJ, Conneely $\mathrm{O}$, Konopleva $\mathrm{M}$ and Andreeff M: HDAC inhibition by SNDX-275 (Entinostat) restores expression of silenced leukemia-associated transcription factors Nur77 and Nor1 and of key pro-apoptotic proteins in AML. Leukemia 27: 1358-1368, 2013.

12. Dear AE, Liu HB, Mayes PA and Perlmutter P: Conformational analogues of oxamflatin as histone deacetylase inhibitors. Org Biomol Chem 4: 3778-3784, 2006.

13. Tan P, Wei A, Mithraprabhu S, Cummings N, Liu HB, Perugini M, Reed K, Avery S, Patil S, Walker P, et al: Dual epigenetic targeting with panobinostat and azacitidine in acute myeloid leukemia and high-risk myelodysplastic syndrome. Blood Cancer J 4: e170, 2014

14. Livak KJ and Schmittgen TD: Analysis of relative gene expression data using real-time quantitative PCR and the 2(-Delta Delta C(T)) method. Methods 25: 402-408, 2001. 
15. Cheson BD, Greenberg PL, Bennett JM, Lowenberg B, Wijermans PW, Nimer SD, Pinto A, Beran M, de Witte TM, Stone RM, et al: Clinical application and proposal for modification of the International Working Group (IWG) response criteria in myelodysplasia. Blood 108: 419-425, 2006.

16. Dail M, Yang L, Green C, Ma C, Robert A, Kadel EE, Koeppen H, Adamkewicz J, Byon J, Woodard J, et al: Distinct patterns of PD-L1 and PD-L2 expression by tumor and non-tumor cells in patients with MM, MDS and AML. Blood 128: 1340, 2016.

17. Coats T, Smith AE, Mourikis TP, Irish JM, Kordasti S and Mufti GJ: Mass cytometry reveals PD1 upregulation is an early step in MDS disease progression. Blood 128: 4296, 2016.

18. Yang H, Bueso-Ramos C, DiNardo C, Estecio MR, Davanlou M, Geng QR, Fang Z, Nguyen M, Pierce S, Wei Y, et al: Expression of PD-L1, PD-L2, PD-1 and CTLA4 in myelodysplastic syndromes is enhanced by treatment with hypomethylating agents. Leukemia 28: 1280-1288, 2014.

19. Azuma T, Yao S, Zhu G, Flies AS, Flies SJ and Chen L: B7-H1 is a ubiquitous antiapoptotic receptor on cancer cells. Blood 111: 3635-3643, 2008.

20. Ball B, Zeidan A, Gore SD and Prebet T: Hypomethylating agent combination strategies in myelodysplastic syndromes: Hopes and shortcomings. Leuk Lymphoma 58: 1022-1036, 2017.
21. Clark CA, Gupta HB, Sareddy G, Pandeswara S, Lao S, Yuan B, Drerup JM, Padron A, Conejo-Garcia J, Murthy K, et al: Tumor-intrinsic PD-L1 signals regulate cell growth, pathogenesis, and autophagy in ovarian cancer and melanoma. Cancer Res 76: 6964-6974, 2016.

22. Lainey E, Wolfromm A, Marie N, Enot D, Scoazec M, Bouteloup C, Leroy C, Micol JB, De Botton S, Galluzzi L, et al: Azacytidine and erlotinib exert synergistic effects against acute myeloid leukemia. Oncogene 32: 4331-4342, 2013

23. Maiso P, Colado E, Enrique MO, Garayoa M, Atadja P Pandiella A and San Miguel JF: Panobinostat (LBH589) a promising new partner for combination with doxorubicin in acute myeloid leukemia. Blood 112: 1638, 2008.

24. Chiappinelli KB, Strissel PL, Desrichard A, Li H, Henke C, Akman B, Hein A, Rote NS, Cope LM, Snyder A, et al: Inhibiting DNA methylation causes an interferon response in cancer via dsRNA including endogenous retroviruses. Cell 162: 974-986, 2015.

25. Roulois D, Loo Yau H, Singhania R, Wang Y, Danesh A, Shen SY, Han H, Liang G, Jones PA, Pugh TJ, et al: DNA-Demethylating agents target colorectal cancer cells by inducing viral mimicry by endogenous transcripts. Cell 162: 961-973, 2015.

26. Dear AE: Epigenetic modulators and the new immunotherapies. N Engl J Med 374: 684-686, 2016. 